\title{
Prevalence of vitamin D deficiency in adult patients admitted to a psychiatric hospital
}

\author{
Dipen Patel, ${ }^{1}$ Manjunath Minajagi ${ }^{1}$
}

BJPsych Bulletin (2018) 42, 123-126, doi:10.1192/bjb.2017.34

${ }^{1}$ Leicestershire Partnership NHS Trust, Leicester, UK

Correspondence to Dipen Patel (dpatel12@nhs.net)

First received 9 May 2017, final revision 19 Nov 2017, accepted 15 Dec 2017

(c) The Authors 2018. This is an Open Access article, distributed under the terms of the Creative Commons Attribution licence (http://

creativecommons.org/licenses/by/ 4.0/), which permits unrestricted reuse, distribution, and reproduction in any medium, provided the original work is properly cited.
Aims and method Vitamin D deficiency is increasing in the general population, and is linked with physical and mental illness. However, evidence on its prevalence in people with mental illness is limited. This study investigated vitamin D deficiency in 104 adult patients admitted to a psychiatric hospital in the UK.

Results Forty-nine per cent were vitamin D deficient (serum $25(\mathrm{OH}) \mathrm{D}<30 \mathrm{nmol} / \mathrm{L}$ ), and a further $42.3 \%$ were vitamin $D$ insufficient $(<50 \mathrm{nmol} / \mathrm{L})$. On admission, $8.7 \%$ of patients were vitamin $D$ sufficient $(>50 \mathrm{nmol} / \mathrm{L}$ ). There were no statistically significant differences in mean serum vitamin $D$ between different subgroups of mental illness.

Clinical implications Vitamin D deficiency is highly prevalent among individuals with severe mental illness admitted to hospital. Assessment and treatment of vitamin $D$ deficiency should be considered in in-patients to protect musculoskeletal health. Further epidemiological and intervention studies are needed to investigate the role of vitamin $D$ in the pathophysiology of mental disorders.

Declaration of interest None.
It has been known for many years that severe vitamin D deficiency increases the risk of metabolic bone disease, particularly rickets and osteomalacia, and that moderate vitamin D deficiency leads to an increased risk of osteoporosis and fractures. ${ }^{1}$ More recently, observational studies have provided growing evidence of an association between vitamin $\mathrm{D}$ deficiency and an increased risk for many chronic diseases, including autoimmune diseases, some cancers, cardiovascular disease, infectious disease and type 2 diabetes. ${ }^{2}$ The serum level of 25-hydroxyvitamin D (25(OH)D) is the most accurate reflection of an individual's vitamin $\mathrm{D}$ status. The National Osteoporosis Society's guidelines define serum 25 (OH)D levels $<30 \mathrm{nmol} / \mathrm{L}$ as deficient, $30-50 \mathrm{nmol} / \mathrm{L}$ as inadequate in some people, and $>50 \mathrm{nmol} / \mathrm{L}$ as sufficient for almost the whole population. ${ }^{3}$ Vitamin D receptors are found in nearly all tissues of the body, including both neuronal and glial cells in the central nervous system and multiple areas of the human brain, including the prefrontal cortex, hippocampus, cingulate gyrus, thalamus, hypothalamus and substantia nigra, many of which have been implicated in the pathophysiology of mental illnesses such as depression and psychosis. ${ }^{4}$ At a molecular level, vitamin D is known to have numerous roles in nervous system health and disease. Animal models have increased our knowledge and understanding of the mechanisms by which vitamin D deficiency affects brain development and its subsequent influence on adult psychiatric and neurological disease. There is evidence that vitamin D has important roles in neurodevelopment, neuroprotection and neuroplasticity, not only by exerting its biological function directly, but also by influencing the expression of genes at a cellular level. ${ }^{5}$ Evidence on its prevalence in people with mental illness, in the UK, is limited. This study investigated the prevalence of vitamin $\mathrm{D}$ deficiency among adult patients being admitted to a psychiatric hospital in the UK, to ascertain how widespread and severe it is among our patients, and to further assess whether there are stronger associations with certain subgroups of illness such as depressive or psychotic disorders.

\section{Method}

This was a cross-sectional study, designed as a pilot, to estimate the prevalence of vitamin D deficiency in patients admitted to a psychiatric hospital, and to ascertain any associations between severity of vitamin D deficiency and severity and subclass of mental illness, in addition to environmental and social demographic factors.

Ethical approval was gained from the National Health Service (NHS) Health Research Authority via the Edgbaston Research Ethics Committee, reference 15/WM/0434. Management permission was sought and gained from the host research and development department at Leicestershire Partnership NHS Trust.

A total of 153 patients were admitted to the general adult wards of the unit, between 17 February 2016 and 23 April 2016, and were eligible for inclusion in the study. One hundred and thirty seven individuals had capacity to 
consent, of which 104 provided informed written consent. Vitamin D levels were requested alongside standard admission blood tests on serum samples collected by venepuncture. Plasma vitamin D levels of serum samples were analysed in the local pathology laboratory, using Siemens Centaur XP analysers with acridinium ester chemiluminescence technology.

Vitamin D deficiency was defined as a serum 25(OH)D level of below $30 \mathrm{nmol} / \mathrm{L}$, insufficiency was defined as a serum 25(OH)D level of $30 \mathrm{nmol} / \mathrm{L}$ or above but less than $50 \mathrm{nmol} / \mathrm{L}$, and vitamin $\mathrm{D}$ sufficiency was defined as a serum 25(OH)D level of $50 \mathrm{nmol} / \mathrm{L}$ or above.

Primary clinical diagnosis using ICD-10 criteria, ethnicity, gender, age and length of stay were subsequently obtained from participants' electronic in-patient medical records.

Data were initially input and analysed using Microsoft Excel, and further descriptive analysis was conducted on IBM SPSS version 20. The Mann-Whitney U test was used to compare means between different samples.

\section{Results}

A total of 104 participants were included in the study; $51 \%$ $(N=53)$ were male and $49 \%(N=51)$ were female. As shown in Table 1, the mean age of the cohort was 40.6 years, and the median age was 39.5 with a range from 18 to 79 . In terms of ethnicity, 76\% $(N=79)$ of participants were White British, 11.5\% $(N=12)$ were British Asian, 5.8\% $(n=6)$ were Black British, 3.8\% $(N=4)$ were of any other White background and $2.9 \%(N=3)$ were of other Asian background.

In the sample studied, mean serum 25(OH)D was 31.9 $\mathrm{nmol} / \mathrm{L}$, and median serum 25(OH)D was $32.0 \mathrm{nmol} / \mathrm{L}$, with a range from $<15 \mathrm{nmol} / \mathrm{L}$ to $81 \mathrm{nmol} / \mathrm{L}$.

There were no statistically significant differences noted in mean serum $25(\mathrm{OH}) \mathrm{D}$ associated with gender, age or primary diagnosis. As shown in Table 2, individuals with a primary diagnosis of schizophrenia were noted to have the lowest mean serum 25(OH)D of $29.5 \mathrm{nmol} / \mathrm{L}$, while those with bipolar affective disorder had the highest mean serum 25 $(\mathrm{OH}) \mathrm{D}$ level of $33.8 \mathrm{nmol} / \mathrm{L}$. Mean serum 25(OH)D was

\begin{tabular}{|c|c|c|}
\hline \multicolumn{3}{|c|}{ Demographic and clinical characteristics of population sample $(N=104)$} \\
\hline \multicolumn{3}{|l|}{ Age, years } \\
\hline Mean & 40.6 & \\
\hline Median & 39.5 & \\
\hline Range & 61 & \\
\hline Minimum & 18 & \\
\hline Maximum & 79 & \\
\hline$\underline{\text { Gender }}$ & Frequency & $\%$ \\
\hline Female & 51 & 49 \\
\hline Male & 53 & 51 \\
\hline Total & 104 & 100 \\
\hline Ethnicity & Frequency & $\%$ \\
\hline White British & 79 & 76 \\
\hline British Asian & 12 & 11.5 \\
\hline Black British & 6 & 5.8 \\
\hline Any other White Background & 4 & 3.8 \\
\hline Other Asian Background & 3 & 2.9 \\
\hline Total & 104 & 100 \\
\hline Primary diagnosis & Frequency & $\%$ \\
\hline$\underline{\text { Depressive episode }}$ & 29 & 27.9 \\
\hline Bipolar affective disorder & 18 & 17.3 \\
\hline Schizophrenia & 18 & 17.3 \\
\hline Personality disorder & 14 & 13.5 \\
\hline Acute or unspecified psychotic disorder & 7 & 6.7 \\
\hline Psychoactive substance-related disorders & 7 & 6.7 \\
\hline Schizoaffective disorder & 5 & 4.8 \\
\hline Anxiety disorder & 3 & 2.9 \\
\hline Delusional disorder & 2 & 1.9 \\
\hline Eating disorder & 1 & 1 \\
\hline Total & 104 & 100 \\
\hline
\end{tabular}




\begin{tabular}{|lccc|}
\hline Table 2 & Mean vitamin D in subgroups & & \\
& $\begin{array}{c}\text { Serum vitamin } \mathrm{D}, \\
\mathrm{nmol} / \mathrm{L}\end{array}$ & $N$ & s.d. \\
\hline Whole sample & 31.9 & 104 & 13.5 \\
\hline Mean & 32 & & \\
\hline Median & 15 & & \\
\hline Minimum & 81 & & \\
\hline Maximum & & & \\
\hline Gender & 32.1 & 51 & 14.1 \\
\hline Female & 31.7 & 53 & 13.2 \\
\hline Male & & & \\
\hline Primary diagnosis & 33.8 & 18 & 17.2 \\
\hline Bipolar affective disorder & 33.5 & 14 & 15.8 \\
\hline Personality disorder & 31.4 & 29 & 9.4 \\
\hline Depressive episode & 29.5 & 18 & 10.3 \\
\hline Schizophrenia & & & \\
\hline Ethnicity & 33.2 & 79 & 13.7 \\
\hline White British & 28.5 & 12 & 13.9 \\
\hline British Asian & 28.8 & 6 & 14 \\
\hline Black British & 23.5 & 4 & 6.2 \\
\hline Any other White background & 28 & 3 & 10.4 \\
\hline Other Asian background & & & \\
\hline
\end{tabular}

higher in participants of White British ethnicity compared with those of other ethnic backgrounds.

As shown in Fig. 1, 49\% $(N=51)$ of participants were vitamin D deficient (serum 25(OH)D $<30 \mathrm{nmol} / \mathrm{L}$ ), and a further $42 \% \quad(N=44)$ were vitamin $\mathrm{D}$ insufficient ( $<50 \mathrm{nmol} / \mathrm{L}) ; 8.7 \%(N=9)$ of participants were vitamin D sufficient $(>50 \mathrm{nmol} / \mathrm{L})$

Although not statistically significant, mean length of stay in participants with vitamin D deficiency $(<30 \mathrm{nmol} / \mathrm{L})$ was 45.4 days, 8 days longer than those without deficiency $(P=0.23)$

\section{Discussion}

To our knowledge, this is the first study to assess prevalence of vitamin D deficiency in patients admitted to a general adult psychiatry unit in the UK. We found a very high prevalence of vitamin D deficiency in patients with mental illness being admitted to psychiatric hospital, with a total of $49 \%$ being deficient and a further $42 \%$ being insufficient in vitamin D.

The reported prevalence in our study was greater than that found in psychiatric in-patient samples in Australia (58\% less than $50 \mathrm{nmol} / \mathrm{L}){ }^{6}$ New Zealand $(74 \%$ less than $50 \mathrm{nmol} / \mathrm{L})^{7}$ and North America (52.3\% less than $\left.50 \mathrm{nmol} / \mathrm{L}\right){ }^{8}$ and in an out-patient sample in Northern Europe (56\% less than $50 \mathrm{nmol} / \mathrm{L})^{9}$

The prevalence of vitamin D deficiency in our study was higher, and the mean serum vitamin D considerably lower, than in the general population in the UK. Results from the National Diet and Nutrition Survey reported a mean

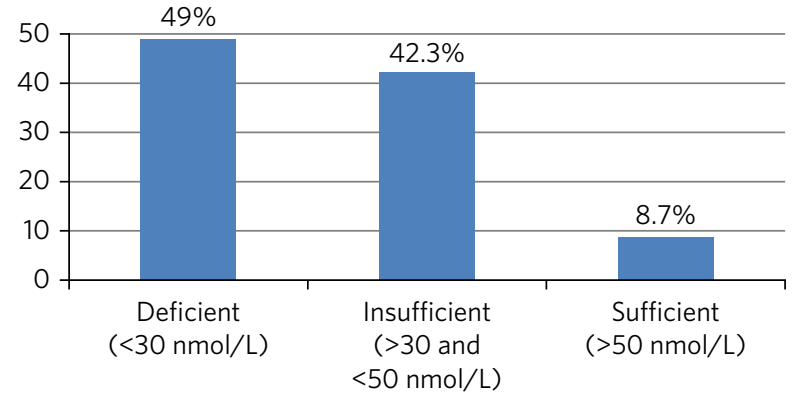

Fig. 1 Percentage of participants found to be vitamin D deficient, insufficient and sufficient $(n=104)$

serum 25(OH)D of $34.8 \mathrm{nmol} / \mathrm{L}$ and a $39.3 \%$ rate of deficiency $(<25 \mathrm{nmol} / \mathrm{L})$ in $19-64$ year olds between the months of January and March, while individuals 65 years and older had a mean serum $25(\mathrm{OH}) \mathrm{D}$ of $40.5 \mathrm{nmol} / \mathrm{L}$ and a $29.3 \%$ rate of deficiency. ${ }^{10}$

In this study, although the differences were not statistically significant, of the four most common diagnostic groups, we found that participants in our sample with a primary diagnosis of schizophrenia had the lowest mean serum vitamin D of $29.5 \mathrm{nmol} / \mathrm{L}$. This finding has been replicated in other studies. A trend towards lower vitamin D levels in individuals with schizophrenia has been reported in a study of psychiatric out-patients, ${ }^{11}$ while a mini meta-analysis of seven studies found that individuals with schizophrenia had a medium effect size for lower vitamin D compared with healthy controls, and also a trend for lower vitamin D levels in comparison with other psychoses. ${ }^{12}$

The UK has an insufficient intensity of ultraviolet sunlight to enable cutaneous synthesis of vitamin D over the winter months, between October and April, thus resulting in the vitamin D status of the UK population peaking in September but then falling continually until the start of the following summer in May, when levels begin to rise again. ${ }^{13}$ Historically, vitamin $\mathrm{D}$ deficiency has been linked to metabolic bone disease, specifically serum concentrations of $<20 \mathrm{nmol} / \mathrm{L}$ being associated with clinical osteomalacia in adults and rickets in children. ${ }^{14}$

It is unclear why the prevalence of vitamin D deficiency found in this study was higher compared with the general population. In addition to cutaneous synthesis during the summer months, vitamin D can be obtained from the diet, but only a few foods such as fatty fish are good sources of vitamin D. ${ }^{1}$ Although institutionalisation in this case would not be a contributing factor, as the participants were newly admitted, it could be hypothesised that people with mental illness are less likely to be spending time outdoors in sunshine and thus produce less endogenous vitamin D over the summer months.

National Institute for Health and Care Excellence and Department of Health guidance identifies the need for vitamin D supplements to be taken by certain at-risk groups, namely all pregnant and breastfeeding women, particularly teenagers and young women; infants and children under 5 years old and people over 65 years old; people who have low or no exposure to the sun, i.e. housebound individuals; and people who have darker skin, for example, 
people of African, African-Caribbean and South Asian origin.

In a change to previous advice, Public Health England revised its guidelines in 2016, based on a report by the Scientific Advisory Committee on Nutrition. The report recognised the growing prevalence of vitamin $\mathrm{D}$ deficiency and is now recommending a reference nutrient intake for vitamin $\mathrm{D}$ of $10 \mu \mathrm{g} /$ day (400 IU/day) throughout the year, for everyone in the general UK population aged 4 years and above, to ensure that the majority of the UK population has enough vitamin $\mathrm{D}$ to protect musculoskeletal health year-round. ${ }^{15}$

Our study has some limitations in terms of its generalisability to the wider population. A total of 104 participants were included, representing a relatively small sample size. Of 153 potential participants, informed consent was obtained from 104 individuals, which could have affected results. As the study was cross-sectional in design, no firm conclusions can be made regarding vitamin $\mathrm{D}$ deficiency, mental illness and the direction of causality if present. Our study was also limited to participants being admitted to a single unit. Finally, the study was conducted during the winter months; thus, the reported prevalence of vitamin D deficiency would be affected by sun exposure if the study was conducted towards the end of summer.

Based on this study, in which almost half of the participants admitted to psychiatric hospital were found to be deficient in vitamin $\mathrm{D}$, assessment and treatment of vitamin $\mathrm{D}$ deficiency by oral supplementation should be considered to protect musculoskeletal health, alongside other physical health interventions, in patients with mental illness admitted to psychiatric wards.

The NHS Five Year Forward View for mental health has recognised the importance of preventable physical health problems in people with severe mental illness, ${ }^{16}$ and thus musculoskeletal health should be optimised where possible.

At the current time, there is insufficient evidence to draw any firm conclusions regarding an association between vitamin $\mathrm{D}$ deficiency and non-musculoskeletal health outcomes, including mental illness. More research in the form of larger epidemiological and intervention studies are needed to investigate the association between vitamin $\mathrm{D}$ and mental health outcomes; indeed, randomised controlled trials are planned that will hopefully shed more light on this intriguing area in the future.

\section{Acknowledgements}

We would like to thank Dr David Clarke, Operational Lead (Research and Development), Leicestershire Partnership NHS Trust, for his invaluable support and guidance.

\section{About the authors}

Dipen Patel is a clinical fellow in Psychiatry and Manjunath Minajagi is a Consultant Psychiatrist at
Bradgate Mental Health Unit, Leicestershire Partnership NHS Trust, Leicester, UK.

\section{References}

1 Lips P. Vitamin D physiology. Prog Biophys Mol Biol 2006; 92(1): 4-8.

2 Wacker M, Holick MF. Sunlight and vitamin D: a global perspective for health. Dermatoendocrinol 2013; 5(1): 51-108.

3 Aspray TJ, Bowring C, Fraser W, Gittoes N, Javaid MK, Macdonald H, et al. National osteoporosis society vitamin D guideline summary. Age Ageing 2014; 43(5): 592-5.

4 Bertone-Johnson ER. Vitamin D and the occurrence of depression: causal association or circumstantial evidence? Nutr Rev 2009; 67(8): 481-92.

5 DeLuca GC, Kimball SM, Kolasinski J, Ramagopalan SV, Ebers GC. Review: the role of vitamin $D$ in nervous system health and disease. Neuropathol Appl Neurobiol 2013; 39(5): 458-84.

6 Berk M, Jacka FN, Williams $U$, Ng F, Dodd S, Pasco JA. Is this D vitamin to worry about? Vitamin $D$ insufficiency in an inpatient sample. Aust $N$ Z J Psychiatry 2008; 42(10): 874-8.

7 Menkes DB, Lancaster K, Grant M, Marsh RW, Dean P, du Toit SA Vitamin D status of psychiatric inpatients in New Zealand's Waikato region. BMC Psychiatry 2012; 12: 68.

8 McCue RE, Charles RA, Orendain GC, Joseph MD, Abanishe JO. Vitamin D deficiency among psychiatric inpatients. Prim Care Companion CNS Disord 2012; 14(2): 11m01230.

9 Humble MB, Gustafsson S, Bejerot S. Low serum levels of 25-hydroxyvitamin D (25-OHD) among psychiatric out-patients in Sweden: relations with season, age, ethnic origin and psychiatric diagnosis. J Steroid Biochem Mol Biol 2010; 121(1-2): 467-70.

10 Bates B, Lennox A, Prentice A, Bates C, Page P, Nicholson S, et al. National Diet and Nutrition Survey Results from Years 1, 2, 3 and 4 (combined) of the Rolling Programme (2008/2009-2011/2012). Public Health England, Food Standards Agency, 2014.

11 Humble MB, Gustafsson S, Bejerot S. Low serum levels of 25-hydroxyvitamin D (25-OHD) among psychiatric out-patients in Sweden: relations with season, age, ethnic origin and psychiatric diagnosis. J Steroid Biochem Mol Biol 2010; 121(1-2): 467-70.

12 Belvederi Murri M, Respino M, Masotti M, Innamorati M, Mondelli V, Pariante $C$, et al. Vitamin D and psychosis: mini meta-analysis. Schizophr Res 2013; 150(1): 235-9.

13 Mavroeidi A, O'Neill F, Lee PA, Darling AL, Fraser WD, Berry JL, et al. Seasonal 25-hydroxyvitamin $D$ changes in British postmenopausal women at 57 degrees $\mathrm{N}$ and 51 degrees $\mathrm{N}$ : a longitudinal study. J Steroid Biochem Mol Biol 2010; 121(1-2): 459-61.

14 Heaney RP. Functional indices of vitamin D status and ramifications of vitamin D deficiency. Am J Clin Nutr 2004; 80(Suppl 6): 1706S-9S.

15 Scientific Advisory Committee on Nutrition. Vitamin $D$ and Health SACN, 2016 (https://www.gov.uk/government/publications/sacnvitamin-d-and-health-report).

16 Independent Mental Health Taskforce. The Five Year Forward View for Mental Health. NHS England, 2016 (https://www.england.nhs.uk/ wp-content/uploads/2016/02/Mental-Health-Taskforce-FYFV-final. pdf). 